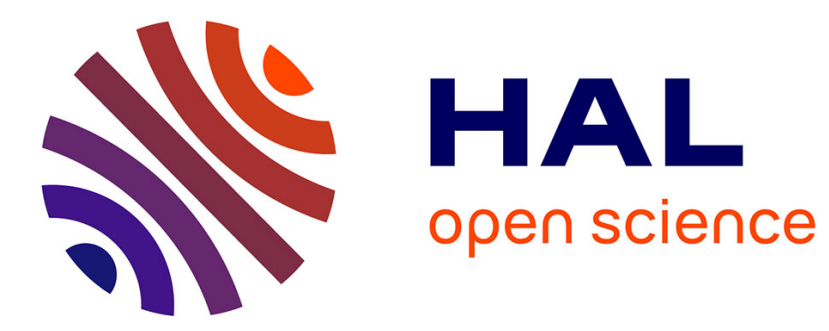

\title{
Differential roles for parietal and frontal cortices in fixed versus evolving temporal expectations: Dissociating prior from posterior temporal probabilities with fMRI Jennifer T Coull, Julien Cotti, Franck Vidal
}

\section{- To cite this version:}

Jennifer T Coull, Julien Cotti, Franck Vidal. Differential roles for parietal and frontal cortices in fixed versus evolving temporal expectations: Dissociating prior from posterior temporal probabilities with fMRI. NeuroImage, 2016, 141, pp.40 - 51. 10.1016/j.neuroimage.2016.07.036 . hal-01471342

HAL Id: hal-01471342

https://hal.science/hal-01471342

Submitted on 20 Feb 2017

HAL is a multi-disciplinary open access archive for the deposit and dissemination of scientific research documents, whether they are published or not. The documents may come from teaching and research institutions in France or abroad, or from public or private research centers.
L'archive ouverte pluridisciplinaire HAL, est destinée au dépôt et à la diffusion de documents scientifiques de niveau recherche, publiés ou non, émanant des établissements d'enseignement et de recherche français ou étrangers, des laboratoires publics ou privés. 
Differential roles for parietal and frontal cortices in fixed versus evolving temporal expectations: dissociating prior from posterior temporal probabilities with fMRI

\author{
Jennifer T Coull*, Julien Cotti, Franck Vidal
}

Laboratoire des Neurosciences Cognitives UMR 7291, Aix-Marseille University \& CNRS, Marseille, France

* Corresponding author:

Laboratoire des Neurosciences Cognitives UMR 7291, Federation 3C, Aix-Marseille University \& CNRS,

3 Place Victor Hugo, 13331 Marseille cedex 3,

France

jennifer.coull@univ-amu.fr

$+33413550942$

Keywords: timing, attention, frontal, parietal, prediction, expectation, foreperiod Running title: Prior and posterior temporal probability 


\begin{abstract}
The ability to predict when an event will occur allows us to respond optimally to that event. Temporal predictability can be either fixed (prior probability) or evolving (posterior probability), in which case it is dynamically updated as a function of the elapse of time itself ("hazard function"). We used fMRI to identify the brain regions involved in either form of temporal prediction, within a single experimental paradigm. Participants performed a cued reaction time (RT) task, in which the target appeared after one of four intervals ("foreperiods") that was either predictable (temporal condition) or variable (neutral condition). As expected, RTs were faster in temporal versus neutral conditions, indicating the behavioural benefit of fixed temporal predictability. RTs also got faster as a function of foreperiod in the neutral, but not temporal, condition, reflecting the evolving temporal predictability of the hazard function. We confirmed that left inferior parietal cortex was preferentially activated by the fixed temporal predictability of temporal (versus neutral) cues. Then, by directly comparing how activity varied as a function of foreperiod in the neutral versus time conditions, we identified the neural substrates of the changes in temporal probability defined by the hazard function, whilst simultaneously controlling for changes related simply to the elapse of time itself. Whole-brain fMRI analyses (independently confirmed by anatomically guided ROI analyses) showed that activity in left inferior parietal cortex tracked the evolving temporal probabilities of the hazard function. ROI analysis further revealed a similar role for right inferior frontal cortex. Our data highlight a key role for left parietal cortex in instantiating the behavioural benefits of temporal predictability, whether predictions are fixed or dynamically evolving.
\end{abstract}

(270 words) 


\section{Introduction}

The ability to anticipate when an event will occur allows us to optimize behaviour and, thus, conserve precious resources. For over a century now, it has been known that reaction times (RTs) are faster when the interval (or "foreperiod") between a warning cue and a target is fixed rather than variable (Woodrow, 1914; Klemmer, 1956).

Nevertheless, even when foreperiods are variable, RTs are significantly faster after long foreperiods than short ones (Woodrow, 1914; Karlin, 1959; Niemi \& Näätänen, 1981). In other words, as the foreperiod increases, so too does the speed of the eventual response. This phenomenon, known experimentally as the "variable foreperiod effect", is traditionally attributed to changes in the probability of target occurrence as a function of the elapse of time. To illustrate, imagine waiting for a bus. The probability of the bus appearing as soon as you arrive at the bus stop is low, but the probability of its appearance increases the longer you wait for it. The longer you wait, the more your expectation grows. This increasing sense of expectancy is driven by the "hazard function": the increasing conditional probability over time that an imperative event will occur, given that it has not already occurred (Elithorn and Lawrence, 1955; Luce, 1986). The hazard function relies on the predictive power of the unidirectional flow of time ("time's arrow"): an event that we expect to occur must do so at some time in the future, given that it has not already occurred in the past. Empirically, this means that the longer we wait for an event to occur, the more certain we are of its occurrence, and the faster we are to respond to it (Niemi \& Näätänen, 1981) or even to perceive it (Vangkilde et al, 2012).

Patients with lesions to the right (Stuss et al, 2005; Vallesi et al, 2007a; Triviño et al, 2010, 2011) or left (Triviño et al, 2010, 2011) lateral prefrontal cortex (PFC) fail to show the typical RT benefits of long foreperiods. Similarly, these benefits are attenuated in healthy volunteers receiving Transcranial Magnetic Stimulation (TMS) to right, though not left, dorsolateral PFC (Vallesi et al, 2007b). fMRI data further confirm that activity in right dorsolateral PFC correlates significantly with the magnitude of the RT benefit afforded by increasing foreperiod length (Vallesi et al, 2009a,b), and peaks at the time of target onset predicted by the hazard function, whether the target actually appears then or 
not (Bueti et al, 2010). In addition, right PFC activity is higher when targets appear after unexpectedly long, rather than unexpectedly short, foreperiods (Coull et al, 2000). Collectively, these data indicate that right lateral PFC is integral in harnessing the temporally predictive information available in the simple passing of time in order to improve performance.

A priori temporal expectations, based on learned temporal associations, can also be used to optimise behaviour. For example, when a traffic light turns amber, we can predict how long it is likely to be before it turns red, allowing us to adjust driving behaviour accordingly (brake or race through). Here, colour acts as a temporally informative cue. This everyday scenario can be simulated in the laboratory by the “temporal orienting" task, an adaptation of the Posner spatial orienting of attention task. In the spatial version of the task, cues indicate whether an impending target will appear on the left or right of the screen (Posner et al, 1980). In temporal orienting tasks by contrast, a symbolic cue informs subjects whether a target is likely to appear after a short or long delay (Coull and Nobre, 1998; Nobre, 2001). Compared to non-informative ("neutral") or misleading ("invalid") cues, temporal cues that correctly predict the time of target onset ("valid" cues) lead to faster (Coull and Nobre, 1998) and more accurate (Correa et al, 2005; Davranche et al, 2011) responses. Neuroimaging studies have revealed a hemispheric lateralisation for temporal versus spatial orienting in left and right parietal cortices respectively (Coull and Nobre, 1998; Coull et al, 2001). Follow-up investigations confirmed that temporal orienting selectively activated left parietal cortex (around the intraparietal sulcus) whether participants used temporal cues to enhance perceptual discriminations or speed motor responses (Davranche et al, 2011), whether responses were left- or right-sided, and whether responses were registered as manual button-presses or ocular saccades (Cotti et al, 2011). Left parietal cortex activation has also been reported when the temporally predictable information used to optimise target detection is not provided by symbolic cues but, instead, by the temporal regularity of a rhythmic visual (Marchant and Driver, 2013) or auditory (Bolger et al, 2014) stimulus stream or a temporally predictable visual trajectory (Assmus et al, 2003; Coull et al, 2008; Merchant et al, 2004). 
Neuroanatomical differences in studies of the variable foreperiod effect on one hand (right lateral prefrontal cortex) and of temporal orienting on the other (left parietal cortex, mostly around the intraparietal sulcus) might reflect functional differences in the way in which temporal predictability is implemented in each paradigm. The temporally predictive information provided by temporal orienting cues (or isochronous rhythms) is fixed and constant: the cue (or rhythm) indicates a priori that the target will appear at a specific moment in time. By contrast, the temporally predictive information provided by the passage of time in variable foreperiod paradigms evolves over time and is updated dynamically. There is no specific moment in time at which the target is expected to appear; rather the probability of target appearance gradually increases as each critical moment is bypassed. In Bayesian terms (Bernardo and Smith, 1994), these two forms of temporal predictability can be considered as equivalent to prior and posterior probability, respectively.

Although prior fMRI studies have investigated the neural substrates of fixed and evolving forms of temporal predictability separately, they have not yet been directly compared. In the current study, we compared the neural substrates of the fixed temporal predictability provided by temporal versus neutral cues to that of the dynamically evolving temporal predictability provided by the passage of time within a single experimental paradigm. Manipulating both forms of temporal prediction within a single paradigm allowed us to identify regions that were activated in common or differentially by these two distinct, but related, processes. In particular, we aimed to substantiate the hypothesis that left parietal cortex is important for initially implementing fixed temporal probabilites a priori, while right prefrontal cortex is involved in dynamically updating these probabilites as a function of the elapse of time (Coull, 2009, 2011, 2015). Importantly, we used a factorial design to control for activity related simply to the passage of time itself by comparing changes over time in neutral versus temporal conditions i.e. a foreperiod $\mathrm{x}$ cue interaction. In the temporal condition, targets were presented after the same foreperiod durations as in the neutral condition, but the conditional probability of their appearance did not increase over time in the same way. Rather than increasing gradually with foreperiod duration, as in the neutral condition, temporal probability in the temporal condition remains constant over time. This factorial 
design ensures that any activity related simply to foreperiod duration would be factored out of a between-condition comparison, pinpointing instead differences in temporal probability. Mento and colleagues recently employed a similar paradigm during EEG investigations in adults (Mento et al, 2015) or children (Mento and Vallesi, 2016). In both studies, the authors found differential modulation of distinct EEG components as a function of temporal and neutral cues. Source localization techniques identified preferential activation of a left-lateralised premotor-parietal network following temporal cues but predominantly prefrontal activity following neutral cues. Unfortunately, the interaction between foreperiod and cue-type was not conducted.

In the current study, temporal cues predicted that the target would appear after one of four different foreperiods whereas neutral cues provided no temporally precise information (Figure 1). We used four foreperiods, rather than only two, so as to track the way in which temporal predictability evolved over time more dynamically than was possible in our previous studies (Coull and Nobre, 1998; Cotti et al, 2011; Davranche et al, 2011; Coull et al, 2013). Sensorimotor factors and foreperiod durations were precisely matched across temporal and neutral cue conditions. In the temporal condition, the probability of target onset time was fixed and constant (100\% certain) across the four foreperiods. In the neutral condition, it changed as a function of foreperiod. We therefore hypothesized that differences in neural activity between the temporal and neutral conditions, averaged across the four foreperiods, would identify regions associated with the behavioural benefits of fixed a priori temporal predictions. On the other hand, we hypothesised that during the neutral condition, in the absence of any other temporal information, participants would rely upon temporally predictive information inherent in the passage of time itself (i.e. the hazard function) to optimise performance. Any change in neural activity as a function of foreperiod in the neutral condition, as compared to the temporal condition (i.e. the cue $\mathrm{x}$ foreperiod interaction), would therefore identify regions selectively involved in the dynamic updating of temporal predictability as a function of time. 


\section{Methods}

\section{Participants}

Sixteen healthy right-handed volunteers (mean age $=26.9$ years; $\mathrm{SD}=5.9$ years), with no record of neurological or psychiatric disorders and normal or corrected-to-normal vision, participated in the fMRI experiment. All volunteers gave written informed consent to the study protocol, which had been approved by the local research ethics committee. The study was performed in accordance with the ethical standards laid down in the Declaration of Helsinki.

\section{Task}

Participants performed a cued visual reaction time (RT) task. The visual cue either predicted the time at which an imminent target would be presented (temporal condition) or acted simply as a trial onset signal, providing no temporally precise information about the time of target presentation (neutral condition). Each trial began with either the temporal or neutral cue, followed by a variable foreperiod (i.e. the interval between the cue and the target) and then brief presentation of an imperative visual target (Figure 1). Participants were informed that they could use the temporal cue to predict the time of target onset, so as to respond to its appearance as quickly as possible. They were told that the neutral cue would not help them to predict target onset but that they should still respond to the target as quickly as possible. Therefore, upon presentation of the cue participants could either predict with $100 \%$ certainty when the target would appear (temporal cue condition) or else could not predict its appearance in advance but could update the likelihood of its appearance as a function of elapsing foreperiod (neutral cue condition). In our experiment, the neutral cue condition is equivalent to the classic “variable foreperiod” paradigm (Woodrow, 1914; Niemi and Näätänen, 1981). Participants were instructed to avoid responding before the target had appeared (i.e. anticipatory responses). Reaction times were calculated as the time between visual presentation of the target and the button-press response.

All stimuli were white and presented in the centre of a black background. A background stimulus, consisting of four white concentric circles, was constantly present 
throughout the task. The temporal cue comprised a brief $(117 \mathrm{~ms})$ brightening of one of the four circles. Brightening of the smallest (innermost) circle indicated that the target would appear after the shortest (533ms) foreperiod, brightening of the next largest circle indicated that it would appear after a $950 \mathrm{~ms}$ foreperiod, brightening of the next largest circle that it would appear after a $1467 \mathrm{~ms}$ foreperiod, and brightening of the largest (outermost) circle indicated that the target would appear after the longest (2083ms) foreperiod. Note that these rather unusual foreperiod durations (533/950/1467/2083ms) were constrained by the screen refresh rate of the monitor: they approximate to our intended foreperiod durations of 500/1000/1500/2000ms. The neutral cue comprised a brief $(117 \mathrm{~ms})$ brightening of all four circles indicating that the target could appear after any one of the four foreperiods (533/950/1467/2083ms) with equal likelihood. The target comprised a cross $(+)$, which was briefly $(117 \mathrm{~ms})$ superimposed over the four circles. Speeded responses to the target were registered with a brief right finger button press. Average trial length was approximately $1.5 \mathrm{~s}$. Inter-trial intervals varied between $2-3 \mathrm{~s}$ (in steps of $\sim 130 \mathrm{~ms}$ ). Temporal and neutral trials were intermixed and the foreperiod varied on a trial-by-trial basis. The combination of the variable inter-trial interval with the variable foreperiod meant that the effective interval between consecutive trials varied between $3.3 \mathrm{~s}-4.9 \mathrm{~s}$.

The two cues (temporal/neutral) and the four foreperiods (533/950/1467/2083ms) constituted a 2 x 4 factorial design. During each of the scanning runs, participants performed a total of 112 trials, comprising 56 temporal cue trials and 56 neutral cue trials (14 trials per foreperiod per cue condition), presented in random order. Across the four scanning runs, this gave a total of 56 trials per foreperiod per condition. Prior to scanning, participants performed a training session to learn the association between the four temporal cues and the four foreperiods. This session began with blocked presentation ( 6 trials) of each of the 4 cue-foreperiod temporal condition combinations. This was followed by a block of 24 temporal cue trials in which the 4 foreperiods were cyclically presented in ascending order of foreperiod duration (from shortest to longest), then a block of 24 temporal cue trials in which the 4 foreperiods were presented in completely randomised order. Finally, participants performed a practice block in which temporal and neutral cue trials (24 trials each) were randomly intermixed. 
Figure 1 about here please

\section{fMRI scanning}

Scans were acquired at the Marseille fMRI centre (http://irmfmrs.free.fr) using a 3-T (3TMAGNEX) Bruker Medspec 30 / 80 Advance whole-body MRI system (Ettlingen, Germany), equipped with a head coil. EPI was used to obtain T2*-weighted fMRI images in the axial plane, using an interleaved slice acquisition sequence. The acquired image volume consisted of $30 \times 4 \mathrm{~mm}$ transverse slices, with an inter scan interval (TR) of $2.0 \mathrm{~s}$. The size of this image volume allowed us to scan the entire cerebral cortex and most of the cerebellum.

Four functional scanning runs, each comprising 234 scans (approximately 8 minutes per run), were acquired for each participant. Run order was counterbalanced across subjects. Within each functional run, the eight trial-types (temporal/neutral cue x 4 foreperiods) were presented in randomised order to ensure optimisation of event-related signal strength (Josephs and Henson, 1999). For 3 of the participants, a failure in stimulus synchronization software meant that data from only three of the four functional runs were usable. A structural MRI was acquired (using a standard T1-weighted scanning sequence, $1 \mathrm{~mm}^{3}$ resolution) to allow anatomically specific localization of significant areas of brain activation.

\section{Data Analysis}

\section{Behavioural Data}

Reaction times (RTs) to detect the target were recorded during the fMRI session and analysed with a repeated measures ANOVA, incorporating cue (time/neutral) and foreperiod (533/950/1467/2083ms) as within-subjects factors. Trials with anticipatory responses and RTs faster than 100ms were excluded from the analysis (9.9\% of all trials). Post-hoc pairwise comparisons were Bonferroni corrected. We also examined the relationship between the RT benefit of time versus neutral cues (RTs in the neutral condition minus RTs in the time condition) to the RT benefit of foreperiod duration (linear regression of RT as a function of foreperiod in the neutral condition minus linear 
regression of RT as a function of foreperiod in the time condition) using Pearsons's correlation.

\section{fMRI Data}

Image processing and statistical analysis of fMRI data were conducted with SPM8 (http://www.fil.ion.ucl.ac.uk/spm/software/spm8). The first 5 images of each run were included to allow for magnetic field saturation and were discarded prior to image processing. All remaining functional images were slice-time corrected using the middle slice in time as reference (slice 27; interleaved acquisition). These images were then realigned to correct for head movement between scans. All functional images were then spatially normalised by matching each image to the standard SPM8 EPI template, resampled to 3-mm isotropic voxel size, and were spatially smoothed using isotropic Gaussian kernels of $8 \mathrm{~mm}$ full-width half-maximum (FWHM).

We then conducted event-related analysis of the preprocessed fMRI data. For each individual participant, stimulus-evoked neural responses were modelled as events with zero duration (delta functions), time-locked to the onset of the cue, and convolved with the canonical hemodynamic response function. We modeled eight separate cue regressors, which represented the factorial combination of cue-type (time/neutral) and foreperiod (533/950/1467/2083ms). We also modelled one regressor of no interest that was time-locked to the onset of the target during both temporal and neutral trials, which accounted for any activity related to execution of a motor response, and six regressors of no interest that modelled the movement parameters derived from the realignment procedure. Images were adjusted for low-frequency physiological drifts, using a highpass filter of 128 seconds, and global changes were removed by proportional scaling.

The general linear model was used to generate statistical parametric maps of the tstatistic, transformed to maps of corresponding $\mathrm{Z}$ values, which provided parameter estimates of event-related activity at each voxel for each experimental condition. At the first level of analysis, each of the 16 participants was analysed separately to define patterns of activation for each comparison of interest for each participant. We defined three main contrasts. First, to identify regions associated with the fixed temporal predictions induced by temporal cueing, we compared activity during the temporal versus 
neutral trials averaged across all foreperiods (i.e. the main effect of cue). This contrast [1 $111-1-1-1-1]$ identified regions whose activity was significantly greater in the temporal condition than the neutral condition. Second, to identify regions whose activity increased as a function of foreperiod duration, we measured how cue-related activity was parametrically modulated by foreperiod duration averaged across both cue conditions (i.e.

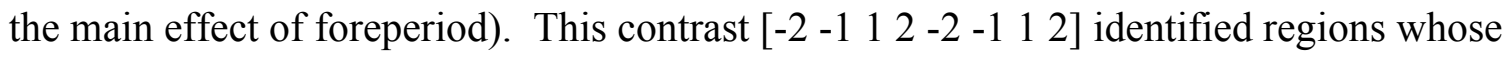
activity significantly increased as foreperiod duration increased in both temporal and neutral trials. Third, to identify regions associated with the dynamic updating of temporal predictions as a function of the passage of time (i.e. the hazard function), we directly compared the parametric modulation of activity as a function of foreperiod in neutral versus temporal trials (i.e. the cue $\mathrm{x}$ foreperiod interaction). This contrast [2 $1-1$

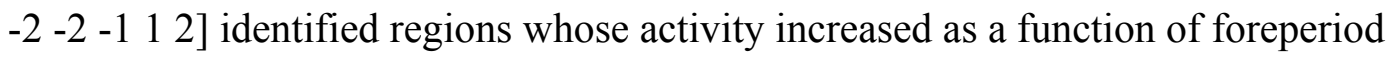
duration in the neutral condition, but not in the temporal condition. By using the interaction term to index the hazard function, we controlled both for foreperiod duration (the same foreperiods were used in both temporal and neutral conditions) and temporal predictability generally (both temporal and neutral conditions induce temporal predictions but temporal predictions evolve dynamically as a function of foreperiod duration in the neutral condition, but remain fixed across foreperiod duration in the temporal condition). The interaction term therefore indexes those regions associated specifically with the dynamic evolution of temporal predictions (i.e. the hazard function) rather than temporal prediction generally.

For each of these three contrasts, the 16 relevant activation maps (one per subject) were entered into a second level of analysis, and statistical inferences for each contrast were derived using one-sample t-tests in SPM. The resulting group-level maps were characterised in terms of both peak amplitude and spatial extent. Statistical maps of significant change in the amplitude of response were initially thresholded at $p<0.001$ (uncorrected for multiple comparisons) at the voxel-level, with the threshold for significance being set at $\mathrm{p}<0.05$, corrected for multiple comparisons (FWE) at the voxel or cluster level. Activations that passed the threshold for significance were either comprised of a significantly large number of voxels (cluster level) or were of a significantly high amplitude (voxel level). In this way, we did not fail to report 
activations that were small in size (and so might fail to pass the cluster level threshold) but strongly activated (and so would pass the voxel level threshold). We report all areas surviving this threshold, which is corrected for interrogation of the entire brain volume. Parameter estimates (beta values) in significant clusters were extracted using the Marsbar region of interest toolbox (Brett et al, 2002) and plotted to aid data interpretation.

To complement the whole-brain analyses described above, and given the strong anatomical hypotheses outlined in the Introduction, we conducted independent ROIbased analyses of activity in left parietal cortex and right prefrontal cortex. We explored how activity changed as a function of experimental condition in AAL-defined left inferior and superior parietal cortices and right inferior (pars triangularis and pars opercularis) and middle frontal gyri. Beta values for each of the eight experimental conditions ( 2 cuetypes x 4 FPs) were extracted from each of the four ROIs using MarsBar (Brett et al, 2002). We conducted repeated measures ANOVAs for each ROI separately, with cue (time/neutral) and foreperiod (533/950/1467/2083ms) as within-subjects factors. We also used Pearson's correlations to explore the relationship between performance and regional activity by correlating the difference in the slope of the RT data in neutral versus temporal trials with the difference in activity in neutral versus temporal trials. We correlated RT slope with activity measured at the longest (2083ms) foreperiod, since this is the time at which the hazard function should exert its greatest influence. ROIs were defined anatomically and independently from the whole-brain analysis, thereby avoiding the error of "double-dipping". 


\section{Results}

\section{Behavioural data}

There were highly significant main effects of both cue condition and foreperiod on RTs. Responses were significantly faster $(\mathrm{F}(1,15)=104.79, \mathrm{p}<0.0001)$ when the target was preceded by a temporal cue $(244 \mathrm{~ms} \pm 11.9)$ rather than a neutral cue $(307.46 \mathrm{~ms} \pm 11.53 \mathrm{~ms})$ and got significantly faster $(\mathrm{F}(3.45)=18.28, \mathrm{p}<0.0001)$ as foreperiod duration increased. Importantly, the main effect of foreperiod was qualified by a significant cue condition $\mathrm{x}$ foreperiod interaction $(\mathrm{F}(3,45)=100.35, \mathrm{p}<0.0001)$, such that RTs got faster as a function of foreperiod following the neutral cue $(\mathrm{F}(3,45)=85.17, \mathrm{p}<0.0001)$ but not following the temporal cue (Figure 2a). Instead, RTs in the temporal condition were actually fastest at the shortest foreperiod $(F(3,45)=7.99, p<0.001)$. Pairwise comparisons at each foreperiod also confirmed results of prior studies showing that the speeding of RTs induced by temporal (versus neutral) cues was evident at the short foreperiods (all $p<0.0001$ at the $533 \mathrm{~ms} / 950 \mathrm{~ms} / 1467 \mathrm{~ms}$ foreperiods) but not at the longest one $(\mathrm{p}>0.1)$ (Coull and Nobre, 1998; Coull et al, 2000; Griffin et al, 2001; Correa et al, 2006).

The RT benefit induced by temporal versus neutral cues was significantly correlated to the RT benefit of foreperiod duration in neutral versus temporal trials $(r=-$ $0.65, \mathrm{p}<0.005)$. Specifically, the more subjects benefitted from the temporal cue, the more they also benefitted from increasingly long foreperiods in the neutral condition (Figure 2b).

Figure 2 about here please

\section{fMRI data}

We used two complementary approaches to analyse fMRI data. First, we interrogated the entire brain volume for regions of activity identified by functionally defined contrasts. Second, we examined patterns of functional change in anatomically defined regions of interest. While the first approach is functionally driven but anatomically impartial, the second approach is anatomically driven and functionally impartial. 


\section{Functionally guided whole brain analyses}

\section{Temporal cueing: fixed temporal predictions}

The main effect of cue condition [temporal-neutral] confirmed prior reports that left parietal cortex is activated significantly more by temporal, than neutral, cueing (Coull and Nobre, 1998; Cotti et al, 2011; Davranche et al, 2011; Coull et al, 2013). We found two distinct clusters of activity in left parietal cortex (Figure 3; Table 1), one in inferior parietal cortex (BA40) and the other located more caudally in superior parietal cortex (BA7). Interestingly, the right-sided homologue of the superior parietal cluster was also significantly more activated by temporal, than neutral, cueing (Figure 3), as was right posterior occipital cortex, left basal ganglia (putamen) and thalamus (pulvinar) (Table 1).

Figure 3 about here please

Table 1 about here please

\section{Foreperiod duration}

The main effect of foreperiod [parametrically increasing foreperiod duration] showed that activity in SMA, dorsal premotor cortex bilaterally, left frontal operculum, left putamen and right lingual gyrus increased with increasing foreperiod (Table 2a).

\section{Hazard function: evolving temporal predictions}

The interaction between cue and foreperiod identified a single cluster of activation in left inferior parietal cortex, located in the intraparietal sulcus (Table 2b), indicating that activity in this region increased as a function of foreperiod more in the neutral condition than in the temporal condition (Figure 4, top panel).

Figure 4 about here please

There were no other clusters of activation that survived the correction for multiple comparisons at the whole brain level. However, given the strong a priori hypothesis that right prefrontal cortex would be activated by the hazard function (Coull et al, 2000; 
Vallessi et al, 2007a,b; 2009a,b; Bueti et al, 2010; Triviño et al, 2010) we conducted a small volume correction (SVC) of this contrast, using a mask comprising AAL-defined right inferior frontal (pars triangularis and pars opercularis) and middle frontal gyri. This SVC analysis revealed a significant cluster of activation in right inferior frontal cortex (Figure 4, bottom panel; Table 2b). We also observed a third cluster of activity in left dorsolateral prefrontal cortex ( $\mathrm{x}, \mathrm{y}, \mathrm{z}$ co-ordinates $=-36,14,25 ; 23$ voxels; $\mathrm{Z}=3.97)$ that did not survive the correction for multiple comparisons but which, given prior reports of bilateral prefrontal involvement in the hazard function (Triviño et al, 2010, 2011), we report here for completeness.

We also examined the cue $\mathrm{x}$ foreperiod interaction in the opposite direction and found that activity in medial occipital cortex ( $\mathrm{x}, \mathrm{y}, \mathrm{z}$ co-ordinates $=15,-82,-8 ; 71$ voxels; $Z=4.18$ ) increased as a function of foreperiod in the temporal condition but not in the neutral condition

Table 2 about here please

\section{Anatomically guided ROI analyses}

\section{Left inferior parietal cortex}

A significant main effect of cue $(F(1,15)=17.60, p=0.001)$ indicated that left inferior parietal cortex was significantly more activated during the time condition than the neutral condition. There was no main effect of foreperiod $(F(3,45)=1.25$, ns) though a significant cue $\mathrm{x}$ foreperiod interaction $(\mathrm{F}(3,45)=3.88, \mathrm{p}=0.015)$ revealed that activity in this region increased as a function of foreperiod duration during the neutral trials $(\mathrm{F}(3,45)=4.55$, $\mathrm{p}<0.01$ ), but remained constant across foreperiod duration during temporal trials $(\mathrm{F}(3,45)=0.14, \mathrm{~ns})$ (Figure 5). Pairwise comparisons at each foreperiod (Bonferroni corrected for multiple comparisons) showed significantly higher activity in the temporal than neutral condition at the $533 \mathrm{~ms}, 950 \mathrm{~ms}$ and $1467 \mathrm{~ms}$ foreperiods (all $\mathrm{p}<0.05$ ). However, there was no difference in activity between temporal and neutral conditions at the $2083 \mathrm{~ms}$ foreperiod. Pearson's correlations revealed a strong relationship between the RT benefits of the hazard function and parietal activity at the crucial longest (2083ms) 
foreperiod $(\mathrm{r}=-0.673, \mathrm{p}<0.005)$. Specifically, the more negative the slope of the RT data in the neutral, versus temporal, condition, the more parietal activity increased in the neutral, relative to temporal, condition (Figure 6a).

\section{Left superior parietal cortex}

A significant main effect of cue $F(1,15)=34.00, p=0.0001$ ) indicated that left superior parietal cortex was significantly more activated during the time condition than the neutral condition (Figure 5). There was no main effect of foreperiod $(\mathrm{F}(3,45)=0.36$, ns), nor cue $\mathrm{x}$ foreperiod interaction $(\mathrm{F}(3,45)=0.80, \mathrm{~ns})$.

\section{Right inferior frontal gyrus}

Although there were no significant main effects of cue $(\mathrm{F}(1,15)=0.29$, ns $)$ or foreperiod $(F(3,45)=1.96, n s)$, a significant cue $x$ foreperiod interaction $(F(3,45)=4.65, p<0.01)$ revealed that activity in right inferior frontal gyrus increased as a function of foreperiod duration during the neutral trials $(\mathrm{F}(3,45)=5.55, \mathrm{p}<0.005)$, but remained constant across foreperiod duration during temporal trials $(\mathrm{F}(3,45)=0.07, \mathrm{~ns})$ (Figure 5). Pairwise comparisons at each foreperiod (Bonferroni corrected for multiple comparisons) showed that the significantly higher activity in the temporal versus neutral condition at $950 \mathrm{~ms}$ $(p<0.05)$ was reversed at $2083 \mathrm{~ms}$, by which point there was significantly higher activity in the neutral than temporal condition $(\mathrm{p}<0.05)$. Pearson's correlations revealed a significant relationship between the RT benefits of the hazard function and frontal activity at the longest $(2083 \mathrm{~ms})$ foreperiod $(\mathrm{r}=-0.538, \mathrm{p}<0.02)$. Specifically, the more negative the slope of the RT data in the neutral, versus temporal, condition, the more frontal activity increased in the neutral, relative to temporal, condition (Figure 6b).

\section{Right middle frontal gyrus}

There were no main effects of cue $(F(1,15)=3.38, n s)$, foreperiod $(F(3,45)=1.17, n s)$, nor cue $x$ foreperiod interaction $(F(3,45)=1.53, \mathrm{~ns})$.

Figure 5 about here please 


\section{Functionally guided ROI analysis}

As a supplementary post-hoc analysis, we defined a smaller ( $3 \mathrm{~mm}$ radius) functional ROI in middle frontal gyrus, centred on the co-ordinates $(\mathrm{x}, \mathrm{y}, \mathrm{z},=52,40,26 \mathrm{~mm})$ provided by Vallesi et al (2009a). Beta values in each experimental condition were extracted from this ROI using MarsBar (Brett et al, 2002) and the pattern of activity across conditions was analysed with a repeated measures ANOVA that included cue (time/neutral) and foreperiod (533/950/1467/2083ms) as within-subjects factors. There were no significant main effects of cue $(F(1,15)=0.42, n s)$ nor foreperiod $(F(3,45)=0.86, n s)$. However, the significant cue $x$ foreperiod interaction $(F(3,45)=3.64, p<0.05)$ indicated that activity in right middle frontal gyrus increased as a function of foreperiod duration during the neutral trials $(\mathrm{F}(3,45)=2.98, \mathrm{p}<0.05)$, but remained constant across foreperiod duration during temporal trials $(\mathrm{F}(3,45)=0.48, \mathrm{~ns})$. 


\section{Discussion}

Being able to predict when an event is going to occur improves sensorimotor processing of that event. Using a cued reaction time (RT) task, we confirmed that both the fixed temporal predictability of temporal cues, and the evolving temporal predictability of the hazard function, significantly speeded RTs. To identify areas linked to different forms of temporal expectation, we interrogated the entire brain volume for regions whose activity significantly changed as a function of cue (temporal/neutral) or the interaction between cue and foreperiod duration. fMRI analyses showed that left inferior parietal cortex, particularly around the intraparietal sulcus, was engaged both by the fixed temporal predictability of temporal cues and by the dynamic updating of temporal probabilities in the neutral cue condition (the hazard function). Right inferior frontal cortex, on the other hand, was engaged by dynamically evolving, but not fixed, prediction mechanisms. The results of these whole-brain analyses were confirmed by independent anatomically and functionally guided ROI analyses.

\section{Behavioural benefits of fixed and evolving temporal predictability}

As predicted, RTs to detect the target were faster after temporal cues than neutral cues, reflecting the behavioural benefits of temporal cueing. RTs were also faster for longer foreperiods, reflecting the behavioural benefits of the hazard function. Importantly, a significant interaction between cue and foreperiod indicated that although RTs got faster as a function of foreperiod in the neutral condition, they remained relatively constant in the temporal condition. These data clearly reflect the way in which the probability distribution evolves over time in the neutral condition: the probability of the target appearing at the shortest foreperiod after a neutral cue was $25 \%$, rising to $33 \%$ at the second foreperiod, then to $50 \%$ at the third, until reaching $100 \%$ probability only at the fourth and longest foreperiod. On the other hand, the probability distribution in the temporal condition was fixed and constant: the probability of the target appearing at the cued foreperiod after a temporal cue was $100 \%$ for all four foreperiods. Consequently, RTs were significantly faster after temporal cues than neutral ones at the three shorter foreperiods, but no different at the longest foreperiod when the probability of target 
appearance was $100 \%$ in both conditions. It could be argued that the speeding of RTs as a function of foreperiod in the neutral condition does not reflect the effects of temporal predictability but, instead, simply reflects the longer period of time available in which to prepare and hone the motor response. However, RTs didn't decrease as a function of foreperiod in the temporal condition, even though foreperiods in this condition afforded the same amount of time to prepare the response. The differential pattern of RTs in the temporal versus neutral condition is therefore more likely to reflect a process that is differentially engaged by the two cue conditions (e.g. fixed versus evolving temporal predictability) than a more fundamental one that is common to both (e.g. time to prepare a motor response).

Incidentally, RTs in the temporal condition were not just invariant across the three longest foreperiods but, collectively, were actually slower than RTs at the shortest foreperiod. This is reminiscent of the pattern observed in fixed foreperiod paradigms in which, by contrast to variable foreperiod paradigms, RTs are slower at longer foreperiods (Klemmer, 1956; Niemi and Naataanen, 1981). Timing estimates (Gibbon, 1977) are more variable for longer durations. Therefore, even when the foreperiod is $100 \%$ predictable (in fixed foreperiod paradigms or the temporal cueing condition of the current experiment) predictions of target onset time will be less accurate at long foreperiods, resulting in slower and more variable RTs (see also Piras and Coull, 2011).

\section{Left inferior parietal cortex activity indexes both fixed and evolving temporal predictions}

Confirming prior fMRI (Coull and Nobre, 1998; Cotti et al, 2011; Davranche et al, 2011; Coull et al, 2013) and source-localised EEG (Mento et al, 2015) results, we found that activity in left lateral inferior parietal cortex was significantly higher in temporal, compared to neutral, cue trials, implicating this region in the performance benefits of fixed a priori temporal predictions. We further demonstrated that activity located slightly more medially, in left intraparietal sulcus, increased as a function of foreperiod in the neutral condition but not in the temporal condition. Since the objective probability of target appearance increases over time in the neutral condition but not in the time condition, we argue that this specific pattern of activity reflects the neural signature of the 
hazard function. By employing exactly the same foreperiods in both time and neutral conditions we controlled for incidental dynamic processes that also vary over time, such as motor preparation, response inhibition or sustained attention. Such processes would be engaged by the time condition as well as the neutral condition, and so are effectively cancelled out within the factorial design. Instead, and as confirmed by the RT data, any changes in neural activity we observed as a function of foreperiod in the neutral condition, but not in the time condition, are likely to reflect temporal expectancies that evolve over time i.e. the hazard function. Providing further support for our findings, the same pattern of activity was found in the left inferior parietal cortex ROI defined by the AAL database (Figure 5) as in the left intraparietal sulcus cluster identified by wholebrain analyses (Figure 4). In the left inferior parietal cortex ROI, activity was significantly higher in the time condition than the neutral condition at the shortest foreperiods. However, as target probability increased over time in the neutral condition, the patterns of activity in time and neutral conditions gradually converged, until meeting at the longest foreperiod at which point the probability of target occurrence was $100 \%$ in both time and neutral conditions. These patterns of brain activity neatly mirror the behavioural data. Indeed, activity in left inferior parietal cortex strongly correlated with the RT benefits of the hazard function.

Whole-brain analysis further revealed that the fixed temporal expectation provided by a temporal cue activated left and right superior parietal cortices, as well as left inferior parietal cortex. In contrast to the pattern of activity in left intraparietal sulcus however, activity in superior parietal cortices did not vary as a function of foreperiod duration. Indeed, ROI analyses allowed us to functionally dissociate distinct forms of temporal expectation in discrete regions of parietal cortex: left inferior parietal cortex, including intraparietal sulcus, was implicated in both fixed and evolving temporal expectations whereas left (and right) superior parietal cortex was engaged by fixed temporal expectations only. This dorsal-ventral distinction for distinct aspects of temporal expectation is reminiscent of Corbetta and Shulman's (2002) scheme for the role of parietal cortex in attention: in this model, superior parietal cortex directs attention to relevant locations or objects in a goal-directed way, whereas more inferior regions of parietal cortex (temporoparietal junction) redirect attention to unexpected events in a 
more stimulus-driven way. Analogously, our data indicate that superior parietal cortex is implicated in the a priori deployment of attention towards a fixed moment in time (goaldirected), whereas inferior parietal cortex and intraparietal sulcus are involved in dynamically reorienting attention to the next possible foreperiod if the target has not yet appeared (stimulus-driven). Alternatively, we may conceptualize these distinct processes in Bayesian terms: they reflect the orientation of attention in time as a function of prior and posterior probabilities respectively.

Although inferior parietal cortex and intraparietal sulcus activations were leftlateralised, superior parietal cortex activations were observed in both left and right hemispheres. Bilateral activation of parietal cortex has previously been reported for the performance benefits of temporal versus neutral cues (Coull et al, 2013) or spatial versus neutral cues (Gitelman et al, 1999). However, when attentional cueing in the temporal and spatial domains were compared directly, Coull and Nobre (1998) found hemispheric lateralization for temporal versus spatial cueing in left and right parietal cortices respectively. These data suggest that both left and right parietal cortices are engaged whenever a selective attentional cue (either temporal or spatial) is compared to a neutral, non-informative cue, but that left and right parietal cortices are preferentially engaged by temporal and spatial attentional cueing respectively. Incidentally, the preferential activation of left inferior parietal cortex by temporal versus spatial cues in this earlier study (Coull and Nobre, 1998) also indicates that this region is implicated in temporal orienting over and above any spatial component of the temporal cue (small circle $=$ short/large circle=long) that might be thought to have led to the parietal activation. Indeed, fixed temporal probabilities activate left inferior parietal cortex even in an entirely non-spatial paradigm employing isochronous auditory rhythms (Bolger et al, 2014).

Overall, our fMRI data indicate a common anatomical substrate for temporal prediction in left inferior parietal cortex, whether the probabilities underlying that prediction are fixed (temporal cues) or evolving (hazard function). Moreover, the RT benefits of temporal cues correlated significantly with the RT benefits of the hazard function, suggestive of a common functional mechanism: the more participants benefitted from the temporal information conveyed by the hazard function the more the benefitted 
from the temporal information conveyed by the temporal cues. Collectively, these data suggest that left inferior parietal cortex is responsible for implementing the response benefits of temporal prediction, however that prediction has been generated. Prior electrophysiological studies in monkeys have shown that parietal activity reflects the accumulation of sensory evidence over time leading to a particular response (Roitman and Shadlen, 2002; Kiani et al, 2008) and, more specifically, that neural firing in parietal cortex mirrors the hazard function (Janssen and Shadlen, 2005). Our whole brain fMRI approach not only confirms that parietal cortex is important in making use of the hazard function to optimize responding but, in addition, reveals that the changing temporal probabilities of the hazard function are also tracked by activity in inferior frontal cortex.

\section{Right inferior frontal cortex is engaged by dynamic updating of temporal predictions over time}

Activity in right inferior frontal cortex increased as a function of foreperiod in neutral, but not temporal, trials, reflecting the performance benefits of the hazard function. These data support previous findings that right lateral frontal cortex is implicated in the dynamic updating of temporal probabilities over time (Stuss et al, 2005; Vallesi et al, 2007b, 2009a; Bueti et al, 2010). Interestingly, the contrast between neutral and temporal trials as a function of foreperiod identified an additional sub-threshold cluster of activity in left prefrontal cortex, lending some support to prior neuropsychological reports that RT benefits of the hazard function depend upon the integrity of both left and right prefrontal cortices (Vallesi et al, 2009a; Triviño et al, 2010; 2011).

Results of our whole-brain analysis were confirmed and supported by independent anatomically defined ROI analyses. Activity in the right inferior frontal gyrus ROI (comprising pars opercularis and pars triangularis) tracked the increasing temporal predictability of target appearance during neutral trials but did not change as a function of foreperiod duration during temporal trials, suggesting that it is implicated specifically in temporal predictability that evolves as a result of the hazard function. Prior neuropsychological investigations of patients with prefrontal lesions, however, indicate that right prefrontal cortex is critical not only for implementing the hazard function but also for the fixed temporal predictions carried by temporal cues (Triviño et al, 2010; 
2011). Although we found that activity in right inferior frontal cortex was significantly higher in the temporal than neutral condition at the $950 \mathrm{~ms}$ foreperiod (though not at the $533 \mathrm{~ms}$ or $1467 \mathrm{~ms}$ foreperiods) this pattern was reversed at the longest $2083 \mathrm{~ms}$ foreperiod. Therefore although right inferior frontal cortex may be engaged more by temporal than neutral cues at short foreperiods, this pattern is reversed at longer ones, thereby effectively cancelling out any differential activity between cue conditions when data are averaged across foreperiod duration. In other words, the role of right inferior frontal cortex in temporal predictability depends critically upon foreperiod duration, strongly suggesting that it reflects the evolving temporal probabilities of the hazard function. Indeed, activity in right inferior frontal cortex significantly correlated with the RT benefits of the hazard function.

The apparently conflicting neuropsychological and fMRI results might be reconciled by distinguishing the ways in which temporal cueing was measured in these studies. In the current fMRI investigation, we compared temporal cues to neutral cues. In the neuropsychological studies of Triviño and colleagues, they compared temporally valid cues to temporally invalid ones (i.e. the target appeared either when expected (valid) or earlier/later than expected (invalid)). While the comparison of temporal to neutral cues indexes the RT benefits of temporal cues, the comparison of valid to invalid cues indexes both the RT benefits of valid cues and the RT costs of invalid cues. Prior fMRI investigation has shown that a direct comparison of temporally invalid to temporally valid cues activates not only left inferior parietal cortex but also a bilateral network of frontal regions, including right prefrontal cortex (Coull et al, 2000). It is therefore conceivable that performance of right prefrontal patients in the neuropsychological studies was underpinned by an effect on the attentional shifting mechanism required by temporally invalid trials, rather than an effect on the performance benefits of temporally valid cues. A neuropsychological study comparing temporal to neutral cues would help resolve this issue.

ROI analyses further revealed distinct patterns of functional activation in discrete regions of right prefrontal cortex. While activity in right inferior frontal gyrus increased as a function of foreperiod duration in neutral but not temporal trials, reflecting the hazard function, there was no significant change in activity of middle frontal gyrus either 
as a function of foreperiod duration nor as a function of temporal versus neutral cues. These data contrast with previous fMRI findings, which localize the neural mechanisms underlying the hazard function to more dorsal regions of prefrontal cortex (Vallesi et al, 2009a; Bueti et al, 2010) than found here. Experimental differences across studies ${ }^{1}$ make it premature to draw definitive conclusions about the specific region of right prefrontal cortex mediating the behavioural benefits of the hazard function. In addition, our ROI analysis was anatomically coarse, with the middle frontal ROI encompassing a large, functionally heterogeneous area of cortex (Figure 5), which may have diluted any activity related specifically to the hazard function. We therefore conducted a supplementary post-hoc ROI analysis of our data, in which the ROI was functionally (rather than anatomically) defined using the co-ordinates provided by Vallesi et al (2009a). Activity in this more limited region of middle frontal gyrus significantly increased as a function of foreperiod duration in neutral but not temporal trials, mimicking the pattern we found in inferior frontal gyrus, and reflecting the evolving temporal probabilities of the hazard function over time.

\section{SMA activity increases as a function of foreperiod duration independently of temporal predictability}

Activity in SMA, premotor cortex bilaterally and left frontal operculum increased as a function of foreperiod duration in neutral and temporal conditions equally. Activity in left putamen also increased as a function of foreperiod duration in both neutral and temporal conditions, though was consistently higher in the temporal condition. These parametric increases in activity during both temporal and neutral conditions indicate that these regions are not specifically implicated in the hazard function: their activity increased as a function of foreperiod duration in the temporal condition, in which predictability remains fixed across foreperiods, as much as in the neutral condition. These findings are at odds with prior fMRI data suggesting that SMA activity specifically tracks the hazard function (Cui et al, 2009; Bueti et al, 2010). In a variable foreperiod

\footnotetext{
${ }^{1}$ In Vallesi et al (2009), fixed versus evolving forms of temporal predictability were presented in separate blocks, whereas in our study fixed and evolving predictabilities were intermixed within a block. In Bueti et al (2010), analyses measured the temporal profile of activity, identifying areas whose activity showed a phasic peak of activity at predictable times. By contrast, in our study we identified more tonic changes in the amplitude of regional activity as a function of increasing predictability.
} 
task with neutral cues, Cui et al (2009) found phasic increases in SMA activity at the moment of target appearance, with SMA amplitude varying as a function of foreperiod duration (i.e. in line with the hazard function). By contrast, when the time of target appearance was explicitly provided by a visual countdown, SMA activity no longer varied as a function of foreperiod. The authors therefore concluded that SMA activity reflected the active updating of temporal probabilities (i.e the hazard function) rather than the simple passage of time. However, by including both neutral and temporal cues in the current study, we have shown that SMA activity varies with foreperiod duration both when participants have prior temporal expectations about target onset time (temporal condition) as well as when they update temporal expectations as a function of time (neutral condition). The main difference between our temporal cue condition and Cui et al's (2009) countdown condition, both of which fully predict when the target will appear, is the need to actively time the foreperiod following a temporal cue but not during a countdown. Taken as a whole, these data suggest that SMA might be indexing the active timing of foreperiod duration - either in order to update probabilities in the neutral cue condition or to predict target onset time in the temporal cue condition. By contrast, in Cui et al's (2009) countdown condition, no active timing was required: participants had simply to wait until the countdown reached zero. This interpretation is consistent with prior fMRI (Wencil et al, 2010; Coull et al, 2015) and single cell electrophysiological (Mita et al, 2009; Merchant et al, 2011, 2013; Crowe et al, 2014) studies showing that SMA activity increases with active or voluntary timing of stimulus duration.

Foreperiod-related activity increases in both temporal and neutral conditions might instead (or also) reflect motor preparation or response inhibition. Indeed, SMA has previously been implicated in motor preparation (Deiber et al, 1999; Cunnington et al, 2002; Hoffstaedter et al, 2013; Rae et al, 2014) and response inhibition (Mostofsky and Simmonds, 2008; Rae et al, 2014), both of which are processes that would be increasingly engaged over the foreperiod as the participant waits to make their response. Alternatively, activity in SMA might simply reflect the time needed to make the motor response: Grinband et al (2011) found that preSMA activity was higher for slow RTs than fast ones. However, our own data argue against this interpretation. SMA activity increased as a function of foreperiod in both neutral and temporal conditions equally, 
despite the fact that RTs got faster with foreperiod duration in the neutral condition but remained constant over foreperiod in the temporal condition. Moreover, SMA became increasingly active as foreperiod duration lengthened, which, in the neutral condition, was actually associated with increasingly shorter RTs. However, our cluster of activation was located in SMA proper whereas that of Grinband et al (2011) was located more rostrally within preSMA, which might explain at least some of the differences in our findings.

In conclusion, our complementary whole-brain and ROI approach has allowed us to differentiate discrete patterns of functional response in distinct brain regions. Fixed temporal expectations established a priori by temporal cues activated left inferior and superior parietal cortices whereas temporal expectations that evolve over time in line with the hazard function activated left intraparietal sulcus and right inferior frontal cortex. This pattern of results supports and extends prior EEG observations of predominantly left-lateralised premotor-parietal activity for temporal cues and predominantly rightlateralised prefrontal activity for neutral cues (Mento et al, 2015; Mento and Vallesi, 2016). By manipulating both temporal cueing and foreperiod duration within a factorial design, we found that left inferior parietal cortex plays a key role in mediating the RT benefits of temporal predictability, whether temporal probabilities are fixed or evolve dynamically as a function of time.

\section{Acknowledgements}

This work was supported by European Collaborative Research Projects in the Social Sciences (06_ECRP_FP009) and the Agence Nationale de la Recherche (ANR- 12BSH2-0005-05). We would also like to thank Bruno Nazarian for practical assistance. These data appeared in abstract form in Coull JT, Cotti J, Vidal F (2014) Increasing activity in left inferior parietal cortex and right prefrontal cortex with increasing temporal predictability: An fMRI study of the hazard function. Procedia - Social and Behavioral Sciences 126: 41-44. 


\section{References}

Assmus A, Marshall JC, Ritzl A, Noth J, Zilles K, Fink GR (2003). Left inferior parietal cortex integrates time and space during collision judgments. Neuroimage 20 Suppl 1: S82-88.

Bernardo JM, Smith AFM (1994) Bayesian Theory. Wiley, New York.

Bolger D, Coull JT, Schön D (2014) Metrical rhythm implicitly orients attention in time, as indexed by improved target detection and left inferior parietal activation. $J$ Cogn Neurosci 26: 593-605

Brett M, Anton J-L, Valabregue R, Poline J-B (2002) Region of interest analysis using an SPM toolbox. 8th International Conference on Functional Mapping of the Human Brain, Sendai, Japan. NeuroImage 16.

Bueti D, Bahrami B, Walsh V, Rees G (2010) Encoding of temporal probabilities in the human brain. J Neurosci. 30: 4343-4352.

Corbetta M and Shulman GL (2002) Control of goal-directed and stimulus-driven attention in the brain. Nature Rev. Neurosci. 3:201-215

Correa A, Lupianez J, Tudela P (2005) Attentional preparation based on temporal expectancy modulates processing at the perceptual level. Psychon Bull Rev 12: 328-334.

Correa A. Lupiáñez J, Tudela,P (2006) The attentional mechanism of temporal orienting: determinants and attributes. Exp. Brain Res 169: 58-68.

Cotti J, Rohenkohl G, Stokes M, Nobre AC, Coull JT (2011) Functionally dissociating temporal and motor components of response preparation in left intraparietal sulcus. Neuroimage 54: 1221-1230

Coull JT (2009) Neural substrates of mounting temporal expectation. PLoS Biol.7: e1000166

Coull JT (2011) Discrete neuroanatomical substrates for generating and updating temporal expectations. In: Dehaene S and Brannon E (eds.) Time and Number in the Brain: Searching for the Foundations of Mathematical Thought. Elsevier.

Coull JT (2015) Directing attention in time as a function of temporal expectation. In: Arthur W. Toga, editor. Brain Mapping: An Encyclopedic Reference, vol. 2, pp. 687-693. Academic Press: Elsevier.

Coull JT and Nobre AC (1998) Where and When to Pay Attention: The Neural Systems for Directing Attention to Spatial Locations and to Time Intervals as Revealed by Both PET and fMRI. J. Neurosci. 18: 7426-7435 
Coull JT, Frith CD, Büchel C and Nobre AC (2000) Orienting attention in time: behavioural and neuroanatomical distinction between exogenous and endogenous shifts. Neuropsychologia 38: 808-819

Coull JT, Nobre AC and Frith CD (2001) The noradrenergic alpha2 agonist clonidine modulates behavioural and neuroanatomical correlates of human attentional orienting and alerting. Cerebral Cortex 11: 73-84

Coull JT, Vidal F, Goulon C, Nazarian B, Craig C (2008) Using time-to-contact information to assess potential collision modulates both visual and temporal prediction networks. Front Hum Neurosci 2: 10.

Coull JT, Davranche K, Nazarian B, Vidal F (2013) Functional anatomy of timing differs for production versus prediction of time intervals. Neuropsychologia 51: 309- 31

Coull JT, Charras P, Donadieu M, Droit-Volet S, Vidal F (2015) SMA selectively codes the active accumulation of temporal, not spatial, magnitude. $J$ Cogn Neurosci 27:22812298

Crowe DA, Zarco W, Bartolo R, Merchant H (2014) Dynamic representation of the temporal and sequential structure of rhythmic movements in the primate medial premotor cortex. J. Neurosci. 34: 11972-11983

Cui X, Stetson C, Montague PR, Eagleman DM (2009) Ready...Go: Amplitude of the fMRI signal encodes expectation of cue arrival time. PLOS Biol. 7: e1000167.

Cunnington R, Windischberger C, Deecke L, Moser E (2002) The preparation and execution of self-initiated and externally-triggered movement: a study of event-related fMRI. Neuroimage 15: 373-385

Davranche K, Nazarian B, Vidal F, Coull JT (2011) Orienting attention in time activates left intraparietal sulcus for perceptual and motor task goals. J Cogn Neurosci 23: 33183330

Deiber MP, Honda M, Ibanez V, Sadato N, Hallett M (1999) Mesial motor areas in selfinitiated versus externally triggered movements examined with fMRI: effect of movement type and rate. J. Neurophys. 81: 3065-3077

Elithorn A, Lawrence C (1955). Central inhibition: some refractory observations. Quart J Exp Psychol 11: 211-220

Gitelman DR, Nobre AC, Parrish TB, LaBar KS, Kim YH, Meyer JR, Mesulam M (1999) A large-scale distributed network for covert spatial attention: further anatomical delineation based on stringent behavioural and cognitive controls. Brain 122:1093-106. 
Griffin IC, Miniussi C, Nobre AC (2001) Orienting attention in time. Front Biosci. 6, $660-671$

Grinband J, Savitskaya J, Wager TD, Teichert T, Ferrera VP, Hirsch J (2011) The dorsal medial frontal cortex is sensitive to time on task, not response conflict or error likelihood. Neuroimage 57:303-311

Hoffsteadter F, Grefkes C, Zilles K, Eickhoff SB (2013) The "What" and "When" of self-initiated movements. Cereb. Cortex 23:520-530

Josephs O, Henson RN (1999) Event-related functional magnetic resonance imaging: modelling, inference and optimization. Phil Trans Roy Soc London B 354: 1215-1228

Karlin L (1959) Reaction time as a function of foreperiod duration and variability. $J$ Exp. Psychol. 58:185-191

Kiani R Hanks TD, Shadlen MN (2008) Bounded integration in parietal cortex underlies decisions even when viewing duration is dictated by the environment. $J$ Neurosci 28:3017-3029

Luce RD (1986) Response Times: Their Role in Inferring Elementary Mental Organization. New York: Oxford University Press.

Marchant, J. L., Driver, J. (2012). Visual and audiovisual effects of isochronous timing on visual perception and brain activity. Cereb Cortex, 23: 1290-1298

Merchant H, Battaglia-Mayer A, Georgopoulos AP (2004) Neural responses during interception of real and apparent circularly moving stimuli in motor cortex and area $7 \mathrm{a}$. Cereb Cortex 14: 314-331

Merchant, H., Zarco, W., Pérez, O., Prado, L., \& Bartolo, R (2011) Measuring time with different neural chronometers during a synchronization-continuation task. Proc. Nat. Acad. Sci. USA 108: 19784-19789

Merchant H, Pérez O, Zarco W, Gámez J (2013) Interval tuning in the primate medial premotor cortex as a general timing mechanism. J. Neurosci. 33: 9082-9096

Mita A, Mushiake H, Shima K, Matsuzaka Y, Tanji J (2009) Interval time coding by neurons in the presupplementary and supplementary motor areas. Nat. Neurosci 12: 502507

Mento G, Vallesi A (2016) Spatiotemporally dissociable neural signatures for generating and updating expectation over time in children: A high density-ERP study. Dev Cogn Neurosci 19: 98-106 
Mento G, Tarantino V, Vallesi A, Bisiacchi PS (2015) Spatiotemporal neurodynamics underlying internally and externally driven temporal prediction: a high spatial resolution ERP study. J Cogn Neurosci. 27:425-439

Mostofsky SH, Simmonds DJ (2008) Response inhibition and response selection: two sides of the same coin. $J$ Cogn Neurosci 20: 751-761

Niemi P, Näätänen R (1981) Foreperiod and simple reaction time. Psychol Bull 89: 133162.

Nobre AC (2001) Orienting attention to instants in time. Neuropsychologia 39: 1317 1328

Piras F, Coull JT (2011) Implicit, predictive timing draws upon the same scalar representation of time as explicit timing. PLoS One 6:e18203

Posner MI, Snyder C, Davidson BJ (1980) Attention and the detection of signals. $J$ Exp Psychol. 109: 160-174.

Rae CL, Hughes LE, Weaver C, Anderson MC, Rowe JB (2014) Selection and stopping in voluntary action: A meta-analysis and combined fMRI study. Neuroimage 86:381-391

Roitman JD, Shadlen MN (2002) Response of neurons in the lateral intraparietal area during a combined visual discrimination reaction time task. J Neurosci. 22:9475-9489

Stuss DT, Alexander MP, Shallice T, Picton TW, Binns MA, Macdonald R, et al (2005). Multiple frontal systems controlling response speed. Neuropsychologia 43: 396-417

Triviño M, Correa A, Arnedo M, Lupiáñez J (2010) Temporal orienting deficit after prefrontal damage. Brain 133: 1173-1185

Triviño M, Arnedo M, Lupiañez J, Chirivella J, Correa A (2011) Rhythms can overcome temporal orienting deficit after right frontal damage. Neuropsychologia 49: 3917-3930

Vallesi A, Mussoni A, Mondani M, Budai R, Skrap M, Shallice T (2007a). The neural basis of temporal preparation: insights from brain tumor patients. Neuropsychologia 45: $2755-2763$

Vallesi A, Shallice T, Walsh V (2007b) Role of the prefrontal cortex in the foreperiod effect: TMS evidence for dual mechanisms in temporal preparation. Cereb Cortex 17: $466-474$

Vallesi A, McIntosh A.R, Shallice T, Stuss DT (2009a) When time shapes behavior: fMRI evidence of brain correlates of temporal monitoring. J Cogn Neurosci. 21: 11161126 
Vallesi A, McIntosh AR, Stuss DT (2009b) Temporal preparation in aging: a functional MRI study. Neuropsychologia 47: 2876-2881

Vangkilde S, Coull JT, Bundesen C (2012) Great expectations: Temporal expectation modulates perceptual processing speed. J. Exp. Psychol.: Hum. Percept. Perf 38:11831191

Wencil EB, Coslett HB, Aguirre GK, Chatterjee A (2010) Carving the clock at its component joints: neural bases for interval timing. J. Neurophysiol. 104: 160-168

Woodrow H (1914) The measurement of attention. Psychol Monogr. 17. 


\begin{tabular}{l|c|c|c} 
Anatomical structure & $\begin{array}{c}\mathbf{x , y , z ~ c o - o r d i n a t e s} \\
(\mathrm{mm})\end{array}$ & $\begin{array}{c}\text { cluster size } \\
\text { (voxels) }\end{array}$ & Z score \\
\hline temporal - neutral & & & \\
left inferior parietal cortex (BA40) & $-48,-34,37$ & 43 & 4.80 \\
left superior parietal cortex (BA7) & $-18,-76,43$ & 171 & 4.65 \\
right superior parietal cortex (BA7) & $18,-79,55$ & 240 & 4.65 \\
right posterior occipital cortex & $36,-91,10$ & 74 & 4.15 \\
left basal ganglia (putamen) & $-27,11,10$ & 84 & 4.37 \\
left thalamus (pulvinar) & $-9,-10,7$ & 45 & 3.97
\end{tabular}

Table 1 - Brain regions preferentially activated by temporal versus neutral cueing, averaged across foreperiod. Activations are significant at $\mathrm{p}<0.05$ corrected for multiple comparisons at the whole brain-level. BA=Brodmann's Area.

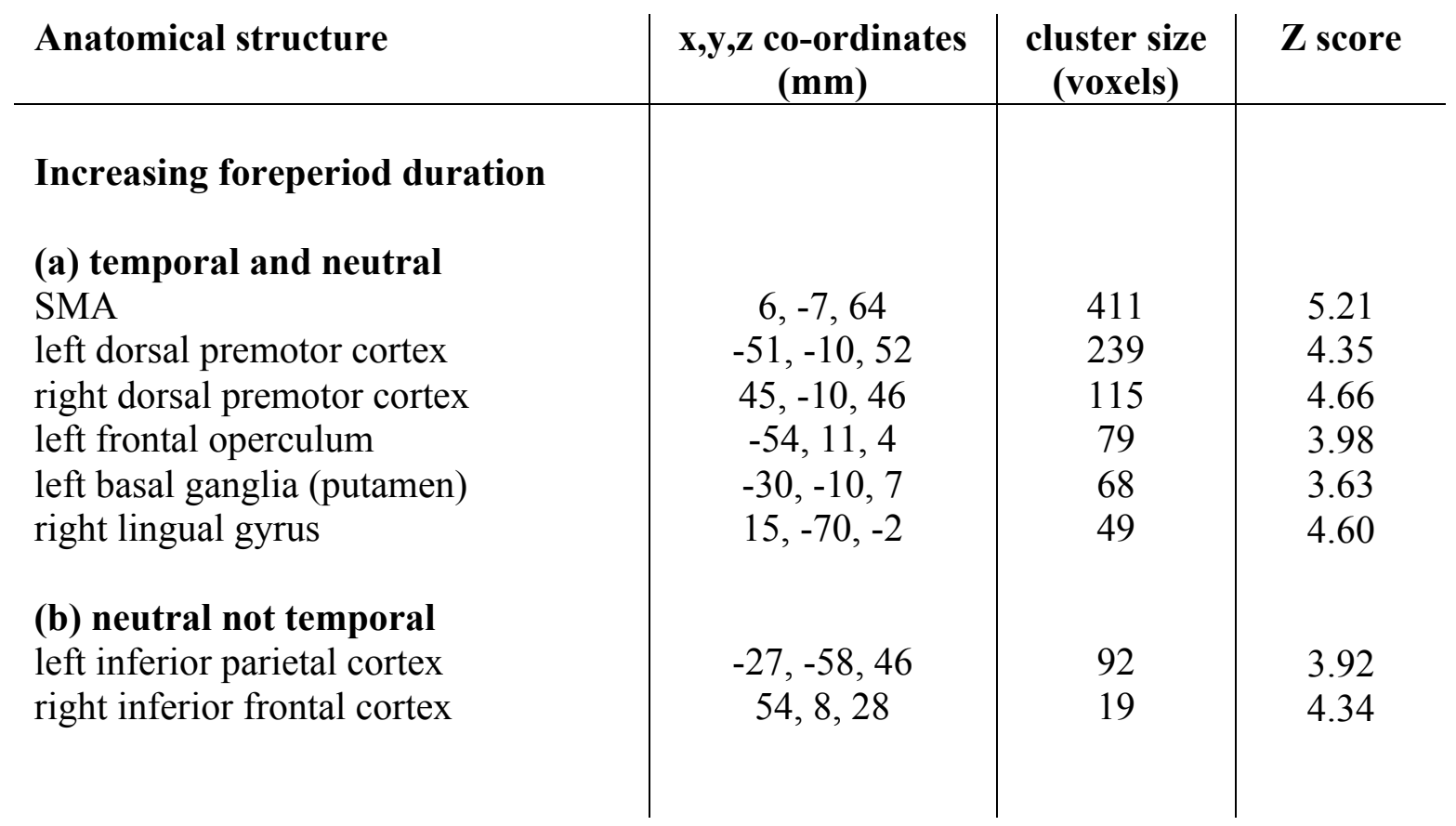

Table 2 - Brain regions whose activity increased parametrically as a function of foreperiod duration. The table shows areas whose activity increased with foreperiod duration either (a) in both temporal and neutral cue conditions or (b) in neutral but not temporal cue conditions. Activations are significant at $\mathrm{p}<0.05$ corrected for multiple comparisons at the whole brain-level or in an anatomically defined region of interest $\left(^{*}\right)$. 


\section{Figure legends}

\section{Figure 1}

Each trial began with presentation of a visual cue, comprising four concentric circles. In the time condition, the size of the highlighted circle predicted the duration of the interval ("foreperiod") between the cue and the target. For example, the smallest circle indicated that the target would be presented after a $533 \mathrm{~ms}$ foreperiod, whereas the largest indicated a $2083 \mathrm{~ms}$ foreperiod. In the neutral condition, all four circles were highlighted, indicating that the target would be presented after a random foreperiod. The target consisted of an upright cross, superimposed upon the background image of the four concentric circles. The participants' task was to detect the appearance of the target as quickly as possible. They were encouraged to use the temporally predictive information conveyed by the temporal cue to improve response speed.

\section{Figure 2}

(a) On average, mean reaction times (RT) were faster following temporal versus neutral cues, reflecting the behavioural benefit of fixed temporal predictions. RTs were faster as a function of increasing foreperiod in the neutral condition, but not in the temporal condition, reflecting the behavioural benefit of the hazard function. Error bars reflect standard errors.

(b) Across participants, the RT benefits of the hazard function (slope of RT data across foreperiod in neutral trials - slope of RT data across foreperiod in temporal trials) correlated significantly with the RT benefit of temporal cues (RT in neutral trials - RT in temporal trials). The more negative the RT slope in neutral trials, the greater the speeding effect of temporal cues. Each point represents an individual participant.

\section{Figure 3}

Compared to neutral cues, temporal cues selectively activated left inferior parietal lobe (IPL) and superior parietal lobes (SPL) bilaterally, across foreperiod (FP) duration. Significant clusters of activation are thresholded at $\mathrm{p}<0.05$ (corrected for multiple comparisons across the entire brain volume) and displayed on a transverse slice of the template brain provided by SPM software. $\mathrm{L}=\mathrm{left}, \mathrm{R}=$ right. Accompanying plots illustrate the mean level of activity (beta values) in significant clusters during temporal (purple) or neutral (blue) cue trials.

\section{Figure 4}

Activity in left intraparietal sulcus (IPS) and right inferior frontal gyrus (IFG) increased as a function of foreperiod (FP) duration during the neutral, but not temporal, condition. Significant clusters of activation are thresholded at $\mathrm{p}<0.05$ (corrected for multiple comparisons across the entire brain volume) and displayed on sagittal and coronal slices of the template brain provided by SPM software. $\mathrm{L}=$ left, $\mathrm{R}=$ right. The accompanying 
plots illustrate the mean level of activity (beta values) in significant clusters during temporal (purple) or neutral (blue) cue trials.

\section{Figure 5}

Four distinct Regions of Interest (ROIs) were defined by AAL and are displayed on a template brain from MRIcron (www.mricro.com/mricron). Activity in left inferior parietal lobe (IPL) and right inferior frontal gyrus (IFG) (ROIs) increased as a function of foreperiod (FP) duration during the neutral, but not temporal, condition. Activity in superior parietal lobe (SPL) was higher in the temporal, versus neutral, condition, independent of FP duration. Activity in middle frontal gyrus (MFG) did not change as a function of either cue or FP. The accompanying plots illustrate the mean level of activity (beta values) in ROIs during temporal (purple) or neutral (blue) cue trials. L=left, $\mathrm{R}=$ right. $*=$ significant $(\mathrm{p}<0.05)$ effect. $\mathrm{ns}=$ non-significant.

\section{Figure 6}

Across participants, the RT benefits of the hazard function (RT slope in neutral trials -RT slope in temporal trials) correlated significantly with increases in activity during neutral versus temporal trials in (a) inferior parietal cortex and (b) inferior frontal cortex. Activity was measured in within the AAL-defined ROIs illustrated in Figure 4. Each point represents an individual participant. ROIs are displayed on an axial slice of the template brain from MRIcron (www.mricro.com/mricron) to complement the views shown in Figure 4. 